\title{
Monte Carlo Modeling of Schottky Contacts on Semiconducting Carbon Nanotubes
}

\author{
H.-Nha Nguyen*, H. Cazin d'Honincthun* ${ }^{\circ}$, C. Chapus*, A. Bournel*, \\ S. Galdin-Retailleau*, P. Dollfus*, N. Locatelli* \\ * Institut d'Electronique Fondamentale, CNRS - Univ. Paris-Sud (UMR 8622), \\ Bât. 220, 91405 Orsay, France \\ ○ Molecular Electronics Laboratory, SPEC, CEA Saclay, 91191, \\ Gif-sur-Yvette, France \\ huu-nha.nguyen@ief.u-psud.fr
}

\begin{abstract}
Metal/carbon nanotube Schottky contacts are studied using particle Monte Carlo simulation. The developed model is based on the WKB approximation and on the Landauer formula. Results are in fairly good agreement with experimental data.
\end{abstract}

\section{Introduction}

Carbon nanotubes (CNT) have recently induced a growing interest in the field of electronic devices. In particular, CNT may be used as channel material in ultimate gate all around nanowire FET because of the extraordinary high mobility observed in CNT [1] and of the compatibility between high $\kappa$ dielectrics and CNT [2]. However, source and drain electrodes of CNTFET are often Schottky contacts.

We have developed a transport model appropriate for studying semiconducting CNT [3] and used our Monte Carlo (MC) device simulator to evaluate performance of CNTFET with ohmic source-drain [4]. In this paper, we present first results related to MC simulation of Schottky contacts on CNT, as schematized in Fig. 1.

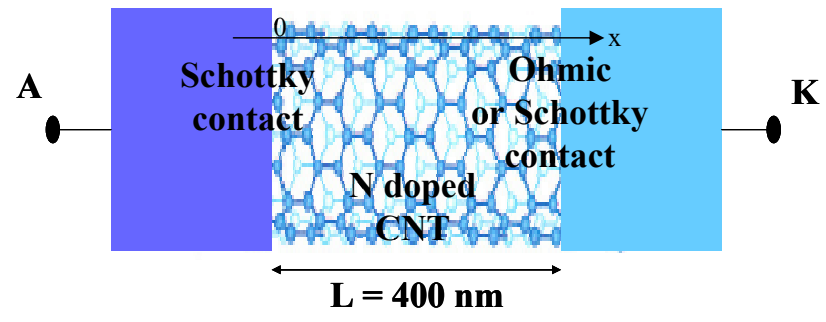

Figure 1: Schema of the studied structure.

\section{Model}

In comparison with the classical $\mathrm{MC}$ technique for ohmic contacts, the treatment of a Schottky contact requires to change the injection from metal to CNT and the particle motion algorithm in the space charge region near Schottky interface. 
After the solution of Poisson's equation at the end of a time step $\Delta t$, which provides the conduction band profile $\mathrm{E}_{\mathrm{C}}(\mathrm{x})$ as plotted in Fig. $2, \mathrm{I}_{\mathrm{inj}} \Delta \mathrm{t} / \mathrm{q}$ particles are injected from metal to CNT where $q$ is the elementary charge. The current $I_{i n j}$ is evaluated from the Landauer formula [6]:

$$
\mathrm{I}_{\text {inj }}=\frac{2 \mathrm{q}}{\mathrm{h}} \int \mathrm{T}(\mathrm{E}) \mathrm{M}(\mathrm{E}) \mathrm{f}(\mathrm{E}) \mathrm{dE}
$$

where $h$ is Planck's constant, $\mathrm{M}(\mathrm{E})$ the number of available subbands in the CNT, and $\mathrm{f}(\mathrm{E})$ the distribution function in the metal. The energy-dependent transmission coefficient $\mathrm{T}(\mathrm{E})$ used in the latter at metal/CNT interface (cf. Fig. 3) is calculated within the WKB approximation. The energy of injected particles is chosen randomly from the bottom of $\mathrm{E}_{\mathrm{C}}$ using a probability deduced from Landauer formula. If $\mathrm{E}$ is higher than the barrier $\Phi_{\mathrm{B}}$, electrons are injected at $\mathrm{x}=0$, otherwise they are injected at position $\mathrm{x}$ where $\mathrm{E}_{\mathrm{C}}(\mathrm{x})=\mathrm{E}$. The initial kinetic energy $\varepsilon$ of tunneling electrons is 0 . For thermionic particles, $\mathrm{E}=\varepsilon+\Phi_{\mathrm{B}}$ is chosen according to the distribution function in the metal.

As for electrons moving in the space charge region, the possibility of tunneling from CNT to the Schottky contact is considered when they "hit" the conduction band $\mathrm{E}_{\mathrm{C}}$, i.e. when $\varepsilon$ vanishes during particle movement. The collection by the metal is effective when a random number $r$ is less than $T\left(E_{C}\right)$. Otherwise, the particle is reflected inside the CNT as in the classical case.

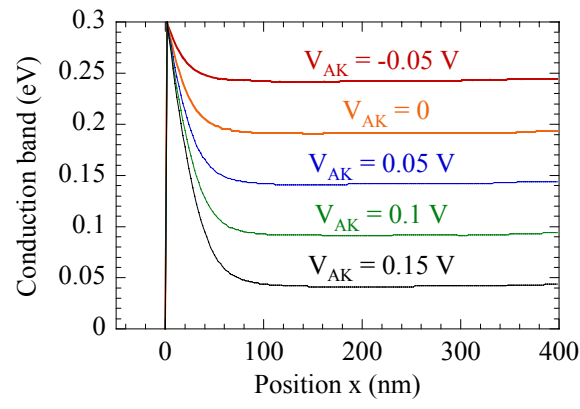

Figure 2: Conduction band along the CNT at different voltages.

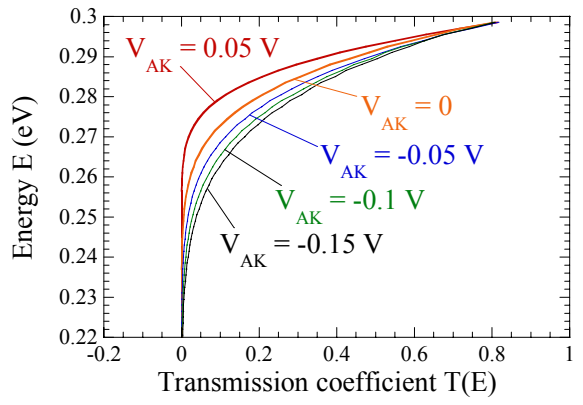

Figure 3: Transmission coefficient as a function of energy.

\section{Results}

In Figs. 4, 5 and 6, we plot I-V curves resulting from the simulation of a $400 \mathrm{~nm}$-long $\mathrm{N}$-doped zig-zag $(16,0)$ CNT with a single Schottky contact. The tunnel effect dominates the current in the reverse regime and in the low bias forward regime. As expected, the current increases when decreasing $\Phi_{\mathrm{B}}$. When increasing the doping level $\mathrm{N}_{\mathrm{D}}$, the current still increases as a result of Schottky barrier thinning. It should be noted that impurity scattering is not included in the simulation. We only consider here the phonon scattering [3,4]. Moreover, the shape of the curves and the current magnitude are in fairly good agreement with experimental results plotted in Fig. 7 [6]. A structure with double Schottky contacts has also been successfully simulated, as shown in Fig. 8. 


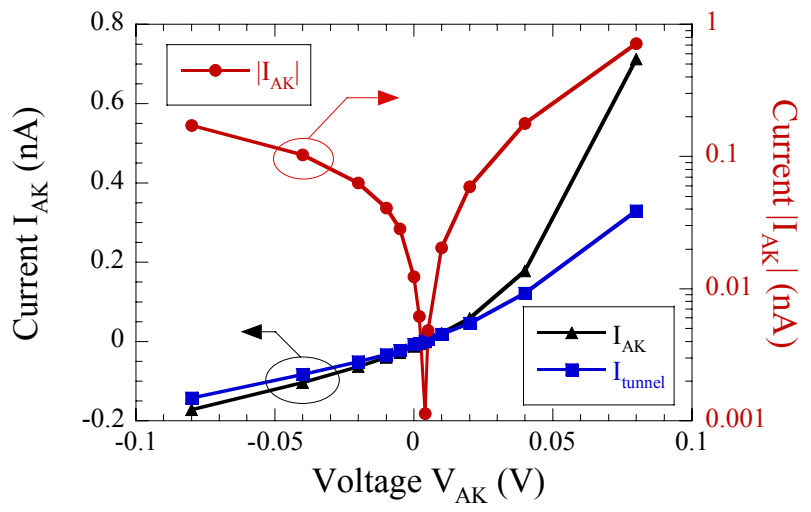

Figure 4: Simulated I-V curve of a single Schottky contact structure for $\Phi_{\mathrm{B}}=0.3 \mathrm{eV}$ and $\mathrm{N}_{\mathrm{D}}=10^{5} \mathrm{~m}^{-1}$.

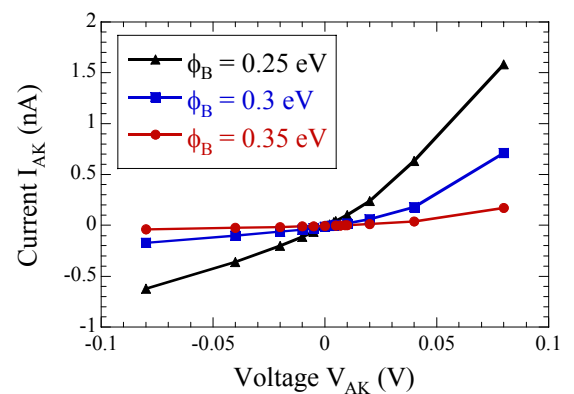

Figure 5: Simulated I-V curves of single Schottky contact structure for $\mathrm{N}_{\mathrm{D}}=10^{5} \mathrm{~m}^{-1}$ and different $\Phi_{\mathrm{B}}$.

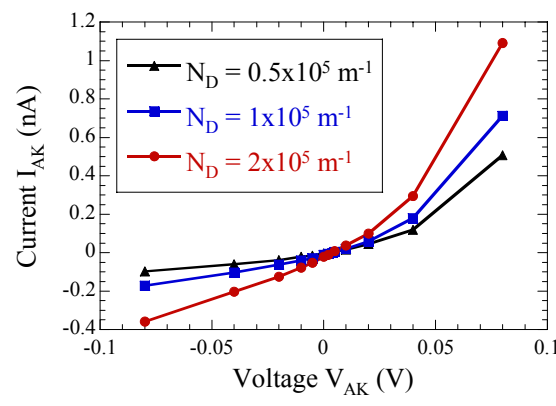

Figure 6: Simulated I-V curves of single Schottky contact structure for $\Phi_{\mathrm{B}}=0.3 \mathrm{eV}$ and different $\mathrm{N}_{\mathrm{D}}$.

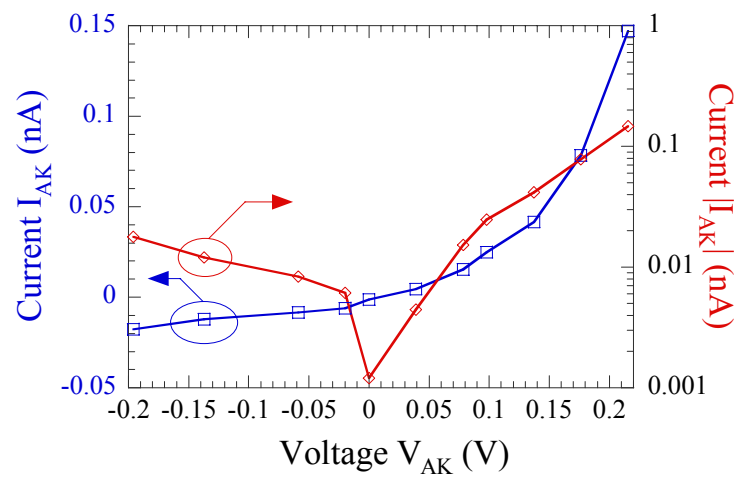

Figure 7: Experimental I-V curve of a single Schottky contact structure from Ref. [6]. 


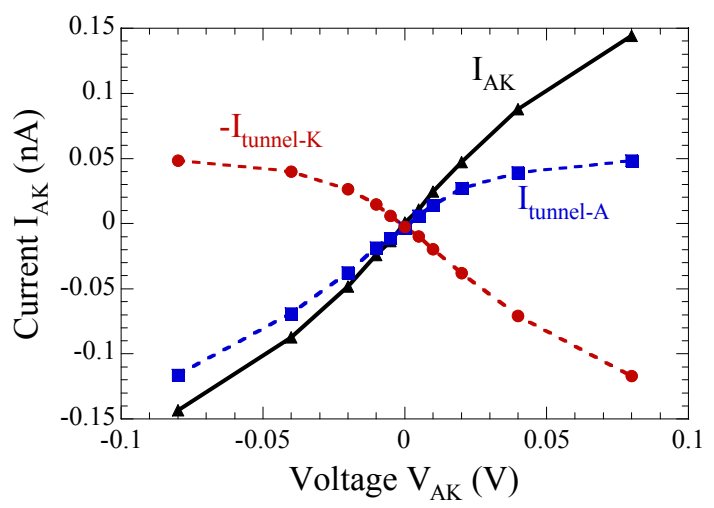

Figure 8: Simulated I-V curve of a double symmetric Schottky contact structure.

$$
\Phi_{\mathrm{B}}=0.3 \mathrm{eV} \text { and } \mathrm{N}_{\mathrm{D}}=10^{5} \mathrm{~m}^{-1} \text {. }
$$

\section{Conclusion}

A correct treatment of Schottky contacts on CNT has been included in a MC device simulator. The model is based on the WKB approximation of the tunnel transmission coefficient and on the Landauer expression of injected current at Schottky contact. This model will be applied to the full simulation of CNTFETs.

\section{Acknowledgements}

This work is partially supported by the French ANR through PNANO HF-CNT and ACCENT projects and by the EC within SINANO Network of Excellence. One of the authors, Huu-Nha Nguyen, thanks the Region Ile de France for a C'Nano grant.

\section{References}

[1] T. Dürkop, S. A. Getty, E. Cobas, M. S. Fuhrer, "Extraordinary mobility in semiconducting carbon nanotubes", Nano Letters, vol. 4, pp. 35-39, 2004

[2] A. Javey, R. Tu, D. B. Farmer, J. Guo, R. G. Gordon, H. Dai, "High performance n-type carbon nanotube field-effect transistors with chemically doped contacts", Nano Letters, vol. 5, pp. 345-348, 2005

[3] H. Cazin d'Honincthun, S. Galdin-Retailleau, J. Sée, P. Dollfus, "Electron-phonon scattering and ballistic behaviour in semiconducting carbon nanotubes", Applied Physics Letters, vol. 87, 172112, 2005

[4] H. Cazin d'Honincthun, S. Galdin-Retailleau, A. Bournel, P. Dollfus, J.-P. Bourgoin, "Influence of gate capacitance on CNTFET performance using Monte Carlo simulation", ULIS 2007 Proceedings, pp. 87-90

[5] S. Datta, "Electronic transport in mesocopic systems", Cambridge University Press, 1995

[6] S. Lu, L. An, Q. Fu, J. Liu, H. Zhang, J. Murduck, "Schottky diodes from asymmetric metal-nanotube contacts", Applied Physics Letters, vol. 88, 133501, 2006 